\title{
A Concept of Value and Sustainable Performance On Affordable High-Rise Residential Decision
}

\author{
Christiono Utomo ${ }^{1, *}$, Suhartono ${ }^{1}$, and Yani Rahmawati ${ }^{2}$ \\ ${ }^{1}$ Institut Teknologi Sepuluh Nopember, Indonesia \\ ${ }^{2}$ Department of Civil \& Environmental Engineering, Universiti Teknologi PETRONAS, Malaysia
}

\begin{abstract}
The need of residential space for low-income people has to be facilitated in urban spatial planning. Because of people will find affordable settlements in the suburbs so their daily mobility will cause congestion. In addition, the inadequate need for a place for low-income households will result in the proliferation of slums in the middle of the city. The goal of this research is to develop an optimal model in generating affordable high-rise residential design performance to improve the environmental quality and sustainability of urban spatial. The object study is a public housing as an affordable housing and formal settlement. When the cost of energy rises and availability decreasing, the need to provided energy-efficient design become more important. It is difficult to quantify and qualify the importance of values. This paper presents value-based decision to select energy system in an affordable high-rise residential building. Criteria for evaluating value are initial cost, life cycle cost, collaborative function, sustainable function, and integrative function. Model formulates, and its implementation based on application of satisficing option on multi criteria decision-making. Commencing at the design and planning stage, the technical, social, and economic sustainability of building energy needs to be considered.
\end{abstract}

\section{Introduction}

The development of urban economy in Indonesia today is quite rapid. These developments resulted in a massive community migration that aims to fill the need for manpower on the various companies. Urban spatial becomes crowded in facilitating the needs that are impacting the city's development. In solving these problems, the government proposed the concept of high-rise residential development for lower-middle-income communities with 1000 tower programs in several cities in Indonesia with several residential types. The realization of the 1000 tower program failed. There are several things behind the failure of the construction of 1000 towers in Indonesia. Tanuwidjaja et al [1] mentioned several factors causing the failure of the 1000 tower program, such as political problems (change of government regime), economic problems (limited funds owned), and environmental problems (development linkage with the sustainability of the surrounding environment). A reference and learning from the failure of the program is the need for consideration of a

${ }^{*}$ Corresponding author: christiono@ce.its.ac.id 
development plan as a whole integrated system. Consideration is made by including or applying the rationale for the sustainability of the built environment in the planning process. Some aspects that need to be considered in the consideration are social, economic and environmental aspects to produce sustainable building design or environmental system.

Stasinopoulos et al [2] introduces one approach that can be applied to the planning process to achieve sustainable development using whole system design (WSD). The method is adopted from the approach in the pre-construction process in the design process. This method has been implemented by the Australian government to achieve a sustainable environment in the country [3]. One of its forms is to educate experts in Australia to apply WSD in designing, with the ultimate aim that the expert considers the sustainability factor of the built environment. So that sustainable development factor becomes the main thinking factor for experts in Australia in planning. In addition to Australia, the Dutch government has also applied the method of approach to development programs in the country [4]. The application of the system is expected the Dutch government can support the development of his country.

This study examines the problems that arise from the economic development of the city through the provision of high-rise settlements devoted to lower-middle-income society by the WSD approach to its planning process, taking into account the social, economic and environmental aspects of developing and building projects to achieve sustainable development. One of the important things in the design process with the WSD approach is to provide a collaborative system facility that can support the achievement of sustainable building design [5]. The complexity of the criteria in the design, especially the design that considers the integration of various aspects into one whole system, leads to the involvement of various experts in the design process with the aim of finding the optimal solution formulation. A good collaborative system is needed to facilitate the diversity of participants involved in the design process [6].

This paper introduces a methodology that can more effectively align the design, development, and performance evaluation with user expectations and economic imperatives. Therefore, the design and development process require expertise from a wide range of disciplines including network architecture, computer-based systems design, economic modeling and business analysis. Over the past few years, significant progress has been made in performance evaluation techniques. Whilst cost is an important consideration, it is only one of the parameters that should influence the decision process and cannot be considered in isolation. If reducing costs results in an inferior solution, then it is possible that this solution will be of significantly less value. In the worst case it may offer no value at all to the original purpose [7]. Therefore, value should be the main consideration when choosing a solution. Value analysis [8] seeks to determine the value of a particular solution, not only in terms of its associated costs but also by taking into account how well it meets the objectives of a particular purpose.

The benefits of this research is optimal design process in generating sustainable building design through an effective design collaboration. It can improve the quality of the surrounding environment, arrangement of urban space with the provision of residential space in high-rise for the community

\section{Conceptual}

\subsection{Value and function analysis}

Value analysis is not concerned with simply minimizing cost. It is possible to increase the value of a product by increasing its function even when this results in greater cost, provided that the added function increases more than the additional cost. The meaning of value may 
be open to interpretation but generally the value of product will judged on some factor such as a high level of performance capability, emotional appeal, style, etc. relative to its cost [9] this can be expressed as:

$$
\begin{aligned}
\text { Value } & =(\text { Performance }+ \text { Capability }) / \text { Cost } \\
& =\text { Function/Cost }
\end{aligned}
$$

Value analysis is a function-oriented methodology that allows a sound understanding of the relationship between the functions of the system and the purpose of their existence to be developed. Decision analysis techniques can then applied to determine the relative value of the alternative solutions for performing the function [10]. This analysis will also take as parameters items such as initial cost, functional performance, reliability, maintenance ability, and quality. A function must be expressed in a measurable parameter in order to obtain a value [11]. Understanding, defining and analyzing functions is an essential factor in the success of a value analysis study. The primary objective of the function analysis is to determine the most beneficial areas for value improvement. The most common approach to classifying functions is to list the physical parts of the project, or steps of a procedure, and attempt to define the functions associated with each part or step. There is no theoretically perfect answer to a function definition problem.

\subsubsection{Collaborative Function}

The main purpose of the design collaboration process is to unite several experts in solving problems and make decisions on the design production process, involving various experts in solving the complexity of the construction field. Patel et al [12] mentions several factors of design success, especially human-related factors. The factors are:

(1) Context: culture, environment, business climate, organizational culture;

(2) Support: tools, networks, resources, training, team building, knowledge management, error management;

(3) Tasks: type, structure, demands;

(4) Interaction process: learning, coordination, communication, decision making;

(5) Teams: roles, relationships, shared awareness, knowledge, common ground, group process, composition,

(6) Individuals: skills; psychological factors, wellbeing;

(7) Overarching factors: trust, conflict, experience, goals, incentives, constraints, management, performance, time.

Participants involved in the process of design collaboration are architects, civil engineer, mechanical and electrical engineer, owners, subcontractors, project managers, technicians and suppliers.

\subsubsection{Integrative Function}

Du et al [13] argues that the individual way of thinking involved in teamwork has to shift and change into a way of thinking that involves and considers the system as a whole thinking, especially in the concept of sustainable development. Consideration of sustainable development aspects became a growing topic, the WSD approach used to achieve these targets also began to be developed by researchers. The main consideration in the WSD approach is the environmental sustainability factor, taking into account the social, economic and environmental sustainability aspects that are integrated with the overall product design. The expected result is the achievement of sustainable development. In realizing the WSD approach and obtaining optimal solutions, various participants with different backgrounds and skills are involved. 


\subsection{Life cycle cost}

The term life cycle cost (LCC) means [14] process for evaluating the total economic worth of a usable project segment by analyzing initial costs and discounted future costs over the life of the project segment. LCC can be implemented at any level of the design process and can also be an effective tool for evaluation of existing building systems. It can be used to evaluate the cost of a full range of projects, from an entire site complex to a specific building system component. It can breakdown the LCC equation into the following three variables: the pertinent costs of ownership, the period of time over which these costs are incurred, and the discount rate that is applied to future costs to equate them with present day costs [15]. As the total cost of ownership including its cost of acquisition, operation, maintenance, conversion, and/or decommission, LCC are summations of cost estimates from inception to disposal for projects. The objective of LCC analysis is to choose the most cost effective approach from a series of alternatives to achieve the lowest long-term cost of ownership. Usually the cost of operation, maintenance, and disposal costs exceed all other first costs. The best balance among cost elements is achieved when the total LCC is minimized. While initial cost is a factor in decisions, it is not the only factor. Four cost driver of energy system of a building was calculated, here salvage value was not calculated because it was not practice in Indonesia. Table 1 presents the LCC and the proportion for each category; initial cost (including investment cost), energy cost, operation and maintenance (O\&M) cost and replacement cost. O\&M cost and energy cost have annual basis.

Table 1. Life cycle cost of affordable high-rise residential building (1000 USD)

\begin{tabular}{lc}
\hline \multicolumn{1}{c}{ Cost category } & Present Worth (1000USD) \\
\hline Initial & 4000 \\
LCC 1: Energy & 250 \\
LCC 2: Operation \& Maintenance & 1500 \\
LCC 3: Replacement & 160 \\
\hline Total Cost (Present value) & 5910 \\
\hline
\end{tabular}

In the calculation of LCC of the residential building, it is essential that the risk and associates with statistical parameters such as discount rate be properly considered. As an affordable building, two most important variables be considered for sensitivity analysis that are discount rate and minimum attractive rate of return (MARR). Sensitivity analysis is a technique for evaluating how stability of a result or outcome varies with variation in various input parameters.

\section{Decision Model Formulating}

There are three main factors supporting the achievement of optimal sustainable design results, namely the WSD approach factor used, the factor of effective design collaboration process, as well as the supporting factors of the concept of environmental sustainability. Each of these factors has criteria and factors supporting the success. In many of the areas to which value analysis has previously been applied the evaluation of alternative solutions has been relatively straightforward. Therefore, in addition to each process that may offer an alternative solution, there are also several possible implementations for each of these modeling and evaluating. By applying value analysis, it can arrive at the solution that offers maximum benefit. A hierarchical approach to evaluation is needed, and it is important to eliminate unsuitable solutions at the highest level of abstraction as possible. 


\subsection{Construction Decision Hierarchy}

The decision hierarchy is a good representation of a problem. It is structured into different components. Fig. 1 presents the model of decision hierarchy. It is based on value model that contains of function and cost. Two of them, that are criteria and sub criteria, are combined into one activity. The goal of the problem $(\mathrm{G}=$ "Value-based selection of building energy system on affordable high-rise residential") is addressed by some alternatives (A = a1; a2; a3). The alternatives should be derived from stakeholder's preferences. The sub-problems, namely cost (C), is consisted of initial cost (capital expenditure) and life cycle cost (LCC). The function (F) is split into three evaluation criteria that will be used to select the best options. The evaluations criteria are written in notation as $\mathrm{c} 1$; $\mathrm{c} 2$; f1; f2; and $\mathrm{f} 3$.

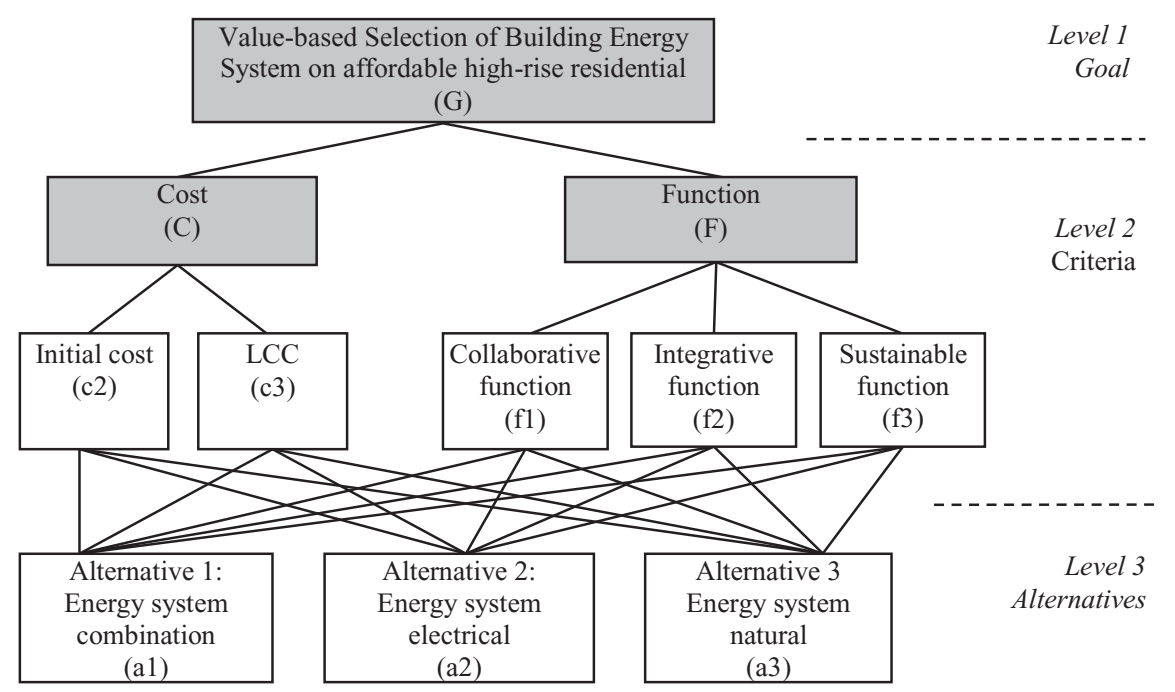

Fig. 1. Model of decision hierarchy

A set of generic process are needed to perform the function. It may be identified as being probable candidates to evaluate the function correctly. It may then be necessary to evaluate before carrying out the final value analysis.

\subsection{Making Judgment}

The judgment and analysis of the decision in this research apply the analytical hierarchy process (AHP) [16]. The scale of pair-wise comparison is the degree of equality from 1 to 9 and reciprocal values $(1 / 9 ; 1 / 8 ; 1 / 7 ; 1 / 6 ; 1 / 5 ; 1 / 4 ; 1 / 3 ; 1 / 2)$. There are two judgments involved in this decision. The first is criteria judgment and the second is technical solution judgment for each criterion. Based on decision hierarchy in Fig. 1, the stakeholders gave their preferences using a set paired comparison questionnaire. Table 2 shows the pairwise comparison. It is judged from decision maker preferences. The result is presented in Fig. 2. It can be observed that the most important evaluation criteria for the goal is 'LCC' followed by 'sustainable function'. Since the decision priority is different for each function, further method can be used for optimization such as goal programming otherwise advanced methods in artificial intelligence [17]. In this study, design by "energy system combination" was decided for the affordable residential building. This decision has been based on the result of qualitative synthesis of LCC analysis and the AHP. 
Table 2. Pairwise comparison among evaluation criteria

\begin{tabular}{lccccc}
\hline $\begin{array}{l}\text { Criteria for energy system } \\
\text { selection }\end{array}$ & $\begin{array}{c}\text { Collaborative } \\
\text { function } \\
\text { (f1) }\end{array}$ & $\begin{array}{c}\text { Sustainable } \\
\text { function } \\
\text { (f2) }\end{array}$ & $\begin{array}{c}\text { Integrative } \\
\text { function } \\
\text { (f3) }\end{array}$ & $\begin{array}{c}\text { Initial } \\
\text { cost }\end{array}$ & LCC \\
(c1) & (c2) \\
\hline Collaborative function (f1) & 1.000 & 5.000 & 0.333 & 7.000 & 0.333 \\
\hline Sustainable function (f2) & 0.200 & 1.000 & 0.143 & 3.000 & 0.143 \\
\hline Integrative function (f3) & 3.000 & 7.000 & 1.000 & 9.000 & 0.500 \\
\hline Initial cost (c1) & 0.143 & 0.333 & 0.111 & 1.000 & 0.111 \\
\hline Life cycle cost (c2) & 3.000 & 7.000 & 2.000 & 9.000 & 1.000 \\
\hline$\lambda$ max=5.365851405 & & $\mathrm{CI}=0.091462851$ & $\mathrm{CR}=0.073760364$ \\
\hline
\end{tabular}

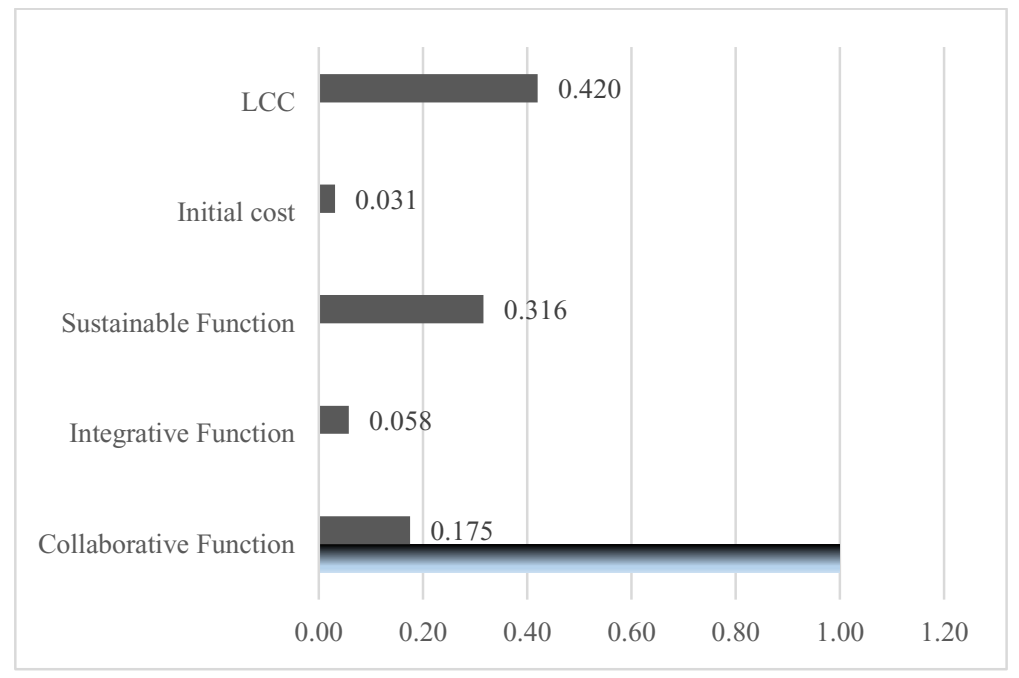

Fig. 2. Weighted of criteria

\subsection{Value-based Analysis (Function/Cost)}

Stirling [18] stated that "A natural procedure of satisficing options is to separate the attributes into two categories, one to involve the attribute that represents functions of an option and the other is to involve attributes that represents losses'. To compare function and cost representing the value of a technical solution, they must be represented on the same scale. This may be done by creating select ability (Ps) and reject ability (Pr) functions and normalizing the problem so that the decision-maker has a unit of function utility and a unit of cost utility to apportion among the options. The columns of (Pr) and (Ps) on Table 3 shows the utility of cost and function for each option of land use solution. Fig. 4 provides a cross plot of function and cost, with Pr the abscissa and Ps the ordinate. It can be seen that the preference value of the decision-makers will impact on the value of the alternative solution [19]. The example given here that a1 is the only has a value greater than $\mathrm{F} / \mathrm{C}=1$ (to select), while the two others decrease to less than $\mathrm{F} / \mathrm{C}=1$ (to reject). 


\section{Weight of each Alternative to Each Criteria}

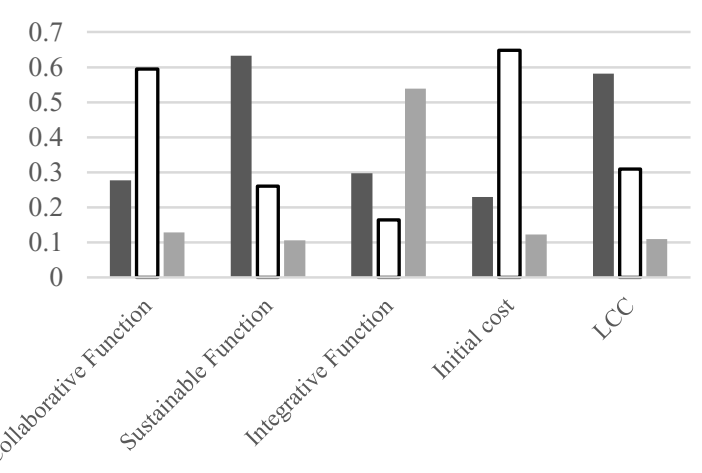

- Combination alternative

口Natural alternative

Electrical alternative

Fig. 3. The best alternatives for each evaluation criteria

Table 3. Value-based options evaluation

\begin{tabular}{|c|c|c|c|c|c|c|c|c|c|c|}
\hline & \multicolumn{5}{|c|}{ Cost } & \multicolumn{4}{|c|}{ Function } & \multirow{2}{*}{$\begin{array}{l}\text { Value }= \\
(\mathrm{Ps}) /(\mathrm{Pr})\end{array}$} \\
\hline & (c1) & (c2) & $\sum$ & Loss & $(\operatorname{Pr})$ & (f1) & (f2) & (f3) & $\sum(\mathrm{Ps})$ & \\
\hline (a1) Combination alternative & 0.01 & 0.24 & 0.25 & 0.05 & 0.11 & 0.05 & 0.04 & 0.09 & 0.180 .33 & 2.96 \\
\hline (a2) Natural alternative & 0.02 & 0.13 & 0.15 & 0.15 & 0.33 & 0.10 & 0.02 & 0.05 & 0.170 .31 & 0.93 \\
\hline \multirow[t]{2}{*}{ (a3) Electrical alternative } & 0.00 & 0.05 & 0.05 & 0.25 & 0.56 & 0.02 & 0.01 & 0.17 & 0.200 .36 & 0.65 \\
\hline & & & & 0.45 & & & & & 0.55 & \\
\hline
\end{tabular}

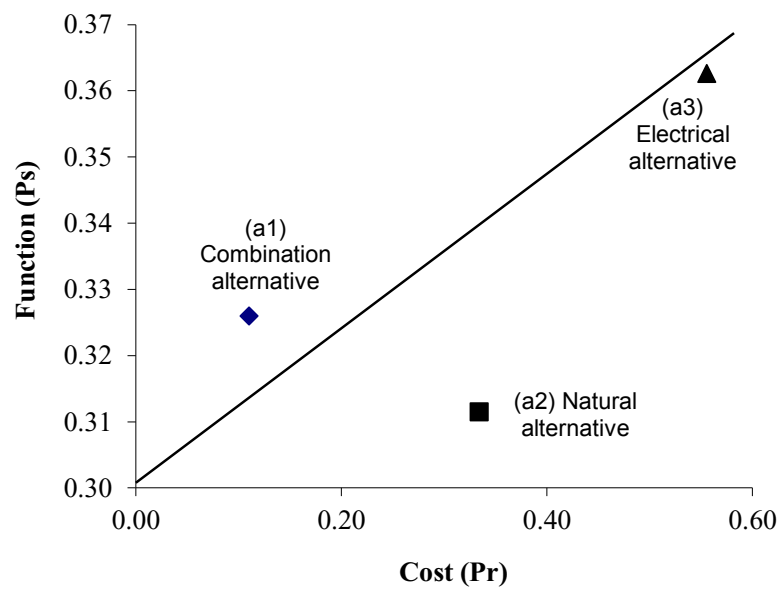

Fig. 4. Value of each alternative solution 


\section{Conclusion}

The implementation results demonstrate a process to select priorities each alternative. Value analysis can help to provide a better alignment between objectives and the choice of design solutions. Life cycle cost and value analysis lead to intellectual effort in the initial design process and an increased active in the evaluation stage. However, given that implementation is the part of the development process that requires the greatest effort, and in many case accounts for the largest proportion of development costs. It believes that this additional effort represents a good investment when compared to the implications of an unsuitable implementation. The framework of methodology for value-based decision is resulted. The methodology consisted of: value-based process and multi-criteria decision process. A value in function/cost is the basis for the methodology. On multi-criteria decision-making, a satisficing option is used by correlating the function and cost to get the value of an alternative solution. Future research is necessary in term of group decision in collaborative process using agreement options and coalition formation.

The authors appreciate the recognition and awards in forms of research grant and fellowship from "Penelitian Berbasis Kompetensi 2018".

\section{References}

1. G. Tanuwidjaja, M.H. Wangsadirja, A. Sudarman. Integrasi kebijakan perencanaan dan desain rumah susun yang berkelanjutan, dalam konteks pembangunan kota yang berkelanjutan (Institut Teknologi Bandung, 2009).

2. P. Stasinopoulos, M. Smith, K. Hargroves, C. Desha, Whole System Design: An Integrated Approach to Sustainable Engineering (London, Eartscan, 2009)

3. The Natural Edge Project, Engineering sustainable solutions program technical design portfolio: whole system design suite (Australian Government: Department of the environment and water resources, 2007)

4. L. Melchert "The Dutch sustainable building policy: a model for developing countries", Journal of Building and Environment, 42, pp. 893-901 (2007)

5. Y. Rahmawati, C. Utomo, N. Anwar, C.B. Nurcahyo, N.P. Negoro, "Theoretical framework of collaborative design issues", Jurnal Teknologi ,70, 7, pp. 47-53, 2014.

6. Y. Rahmawati, C. Utomo, N. Anwar, P. Setijanti, C. B. Nurcahyo, “An empirical model for successful collaborative design towards sustainable project development", Journal of Sustainable Development ,7, 2, pp. 1-14, 2014.

7. A. Idrus, and C. Utomo, "Cooperative Coalition Formation on Value-based Decision", International Symposium on Information Technology - System Development and Application and Knowledge Society ITSim'10, pp. 1114-1118 (June 2010)

8. R. Woodhead, J. McCuish, achieving results: how to create value (Thomas Telford, UK, 2002)

9. J. Kelly, S. Male, D. Graham, Value management in design and construction: the economic management of projects (Blackwell Science, London, 2004)

10. R. J. Wixson, Function analysis and decomposition using function analysis system technique, Proceedings of INCOSE (1999)

11. C. Utomo and A. Idrus, "Life cycle cost and function analysis in value-based design decision" Life-Cycle Civil Engineering - Proceedings of the 1st International Symposium on Life-Cycle Civil Engineering (2008) 
12. H. Patel, M. Pettitt, and J.R. Wilson "Factors of collaborative working: a framework for a collaboration model", Journal of Applied Ergonomics 43, 1-26 (2012)

13. J. Du, S. Jing, L. Liu, "Creating shared design thinking process for collaborative design", Journal of Network And Applications (2011)

14. W. J. Fabrycky, B.S. Blanchard, Life-cycle cost and economic analysis (Prentice-Hall, Englewood Cliffs, 1991)

15. P. Barringer, A life cycle cost summary. International Conference of Maintenance Societies (2003)

16. T.L. Saaty, "Decision making: the analytical hierarchy process and network process (AHP/ANP)" Journal of System Science and System Engineering 13,1: 1-34 (2004)

17. C. Utomo, and Y. Rahmawati, "Knowledge and protocol on collaborative design selection", IEEE Conference on Information Technology, Computer and Electrical Engineering, Semarang, 7-8 Nov, pp. 199 - 202 (2014)

18. W.C. Stirling, Satisficing Games and Decision Making with Applications to Engineering and Computer Science (Cambridge University Press, Cambridge, UK, 2003)

19. C. Utomo, A. Idrus, M. Napiah, M.F. Khamidi, "Aggregation and Coalition Formation on Value-based Decision", In Proceeding of the Symposium on Computational Intelligence in Multi-criteria Decision-Making (MCDM). Nashville - USA, 30 March 2 April: IEEE. p. 118-125 (2009) 